\title{
The Relationship between Allergic Pollinosis and Low Salivary Levels of Secretory IgA in Adults
}

\section{Tetsuro Yamamoto ${ }^{1}$, Tadao Enomoto ${ }^{2}$, Akira Tsuda ${ }^{3}$, Hisayoshi Okamura $^{4}$, Tatsuya Honda ${ }^{1}$, Shaw Watanabe ${ }^{5}$ and Hideyo Yamaguchi ${ }^{1 *}$}

${ }^{1}$ Research Center, TTC Co., Ltd., Tokyo, Japan

${ }^{2}$ NPO Japan Health Promotion Supporting Network, Wakayama, Japan

${ }^{3}$ Department of Psychology, Kurume University, Kurume, Japan

${ }^{4}$ Cognitive and Molecular Institute of Brain Diseases Kurume University, Kurume, Japan

${ }^{5}$ LifeScience Promoting Association, Tokyo University of Agriculture, Tokyo, Japan

*Corresponding Author: Hideyo Yamaguchi, Research Center, TTC Co., Ltd.,

Tokyo, Japan
Received: September 23, 2020

Published: October 07, 2020

(C) All rights are reserved by Hideyo

Yamaguchi., et al.

\section{Abstract}

Secretary IgA is a key element of intestinal immunity. Low levels of secretory IgA (SIgA) and development of allergy in infants were often reported, but it is not in adults. Saliva and serum were simultaneously analyzed from 20 adult patients with Cedar pollinosis aged 22-53 years and 22 non-allergic control adults aged 25-65 years. The mean salivary level of SIgA for JCP patients was significantly lower than the value for control subjects $(58.1 \pm 8.8 \mathrm{ug} / \mathrm{ml}$ vs. $68.6 \pm 12.1 \mathrm{ug} / \mathrm{ml})$. On the contrary, Cedar pollen specific IgE in the serum was $11.5 \mathrm{UA} / \mathrm{ml}, 4.05,23.1$, respectively (median, $25^{\text {th }}-75^{\text {th }}$ percentile) in pollinosis group, while it was $<0.34 \mathrm{UA} / \mathrm{ml}$ (detection limit) in control group. Low levels of salivary SIgA in adults was associated with increased allergic manifestations.

Keywords: Allergic Pollinosis; Japanese Cedar Pollen; Saliva; Secretory IgA, Specific IgE Antibodies

\section{Introduction}

Immunoglobulin A (IgA) is the predominant immunoglobulin isotype in humans. Although accounting for only 10 - 15\% of the total serum immunoglobulins, IgA is quantitively the most important immunoglobulin in mucous secretions [1]. Most of the $\operatorname{IgA}$ existing in tears, saliva, respiratory secretions, milk and digestive tract fluids is present as secretory IgA (SIgA), consisting of dimeric IgA, a joining molecule called J chain and a glycoprotein called secretory component [2] SIgA is produced locally in mucosal tissues and glands by mucosa-associated lymphoid tissues (MALT), as well as by the gut-associated lymphoid tissues (GALT) including Payer's patches in the distal small intestine [3]. Although the primary function of GALT is to protect the gut, it also acts as the main effector of site for mucosal protection for other parts of the body, such as the nasal cavity and conjunctiva $[3,4]$.
The SIgA immune response in mucosal secretions has a welldefined role in limiting not only the passage of infectious microorganisms, but also that of potentially immunogenic macromolecules through the mucous barrier. SIgA may block adherence and penetration of antigens through the mucosal epithelium. In this regard, it has been proposed that a deficiency in total SIgA and/or specific IgA antibodies in mucosal secretions can function as a predisposing factor in the development of allergy [5-7].

Thus, there is a rationale to consider that high levels of total and/or allergen-specific SIgA in mucosal secretions could prevent allergen absorption and thereby sensitization and subsequent development and attack of allergy. This possibility is supported by the results from the studies conducted on neonates or infants demonstrating that low levels of SIgA in saliva are associated with an increased risk for allergy [8-11]. However, this is controversial 
because a tendency towards the opposite has also been reported [12]. Another issue generated is that almost all data on a possible protective role of SIgA against development of allergy have been obtained for newborns or young children ( $<4$ years) and much fewer data are available for the adult population.

Japanese cedar pollinosis (JCP) is the most common seasonal allergic pollinosis in Japan caused by inhalation of the pollen of the Japanese cedar (Cryptomeria japonica). The prevalence of JCP is estimated to be as high as $26.5 \%$ of the Japanese population and $54.1 \%$ of patients with JPC are treated with prescribed anti-allergic drugs [13]. The disease develops various nasal and eye symptoms, including sneezing, itchy nose and/or throat, rhinorrhea and itchy, watery and/or red eyes, resulting in interference with daily activities and deterioration of quality of life. For these reasons, JCP generates a major public health problem in both infant and adult populations.

The present study was attempted to investigate the possible relationship between SIgA insufficiency and manifestation of JCPassociated allergic symptoms and/or production of specific IgE antibodies in the adult population. For this purpose, we retrospectively analyzed the salivary level of total SIgA, along with the serum levels of specific IgE and total immunoglobulins, in adult patients with JCP (allergic pollinosis) and non-allergic healthy adult individuals to compare between the two populations.

\section{Materials and Methods}

Subject

Informed consent was obtained from all subjects from whom saliva samples and blood samples were collected. The subjects consisted of 20 adult patients with JCP and 22 non-allergic control adult individuals. JCP patients, six males and 14 females, age range 22 - 53 (mean, 42.0) years, were those who had two-year or longer clinical history of JCP and met two or more of the following criteria: (i) positive skin test and/or positive specific IgE test for Japanese cedar pollen allergen; (ii) eosinophil-rich nasal discharge; and (iii) positive reaction to nasal challenge with Japanese cedar pollen allergen. Control healthy individuals, six males and 16 females, age range 25 - 65 (mean, 37.9) years, who have had no history of JCP or any other allergic disease, were recruited at random.

\section{Saliva and blood sampling and preparation}

Saliva samples were collected from all of the 42 subjects during the time period when Japanese cedar pollens were not dispersed. At the same time-point, blood samples were drawn from all subjects.
To collect saliva samples, subjects were asked to rinse their mouths with water and to rest for 15 min during which eating and smoking were prohibited. Then each subject took a cylinder-formed cotton pad out of "Salivatte" (SARSTEDT AG and Co., Germany), a saliva collection vial, and bited it lightly with his/her molar teeth. The cotton pad was further subjected to a light biting and rolling in the mouth so as to be impregnated with a sufficient amount of saliva. After two minutes, the cotton pad was taken out from the subject's mouth, put in the vial, sealed therein by capping, and then frozen to be kept until use. Blood samples drawn were allowed to clot at room temperature, and the sera were aliquoted and stored at $-70^{\circ} \mathrm{C}$ until analysis.

\section{Measurement of SIgA in saliva}

For measurement of SIgA, "EIA SIgA Test” (MBL, Ina-shi, Japan), which enables detection of only SIgA, was used. Each saliva sample diluted 1:40 in the indicated buffered solution was incubated at $37^{\circ} \mathrm{C}$ for $1 \mathrm{~h}$ with an anti-human secretory component-solidified porous polystylene ball in a tube. After washing with PBS, the tube receiving the reaction mixture was incubated at room temperature (ca. $20^{\circ} \mathrm{C}$ ) for $1 \mathrm{~h}$ with $0.3 \mathrm{ml}$ of peroxidase-conjugated anti-human IgA rabbit IgG/Fab'. After washing with PBS, antibody binding to the polystyrene ball was detected by addition of $0.5 \mathrm{ml} /$ tube of 0 phenylenediamine used as substrate. Human SIgA was diluted in 6 steps for standard curve. The detection intervals were 0.06 to $64 \mu \mathrm{g} / \mathrm{mL}$ for human SIgA.

Measurement of Japanese cedar pollen allergen-specific IgE antibodies in serum

Immunoglobulin E antibodies against Japanese cedar pollen allergen (specific IgE) in serum samples were assayed by the fluorescent EIA technique (CAP.RAST) with the use of a commercial kit "Unicap-specific IgE” (Phadia Japan, Tokyo). A Japanese cedar pollen allergen-containing plastic capsule ("immuno CAP") used for the test was the product of Phadia Japan, Tokyo. Serum levels higher than $0.35 \mathrm{UA} / \mathrm{mL}$ were considered as CAP-RAST-positive and those lower than the value as CAP-RAST-negative.

\section{Measurement of total immunoglobulins in serum}

EIA-based measurements of total IgA, IgG and IgE in serum were performed with the use of "N-Assay TIA IgA-SH Nittobo" (Nittobo Medical Co., Tokyo), "N-Assay TIA IgG-SH Nittobo" (Nittobo Medical Co., Tokyo) and "Unicap Total IgE” (Phadia Japan, Tokyo), respectively. All the tests were carried out according to the manufacturer's instructions. 


\section{Statistics}

All statistical analyses were performed on the logarithms of immunoglobulin levels since immunoglobulin concentrations follow a lognormal rather normal frequency distribution [14]. Means and standard deviations (SDs) were performed on the nonlogtransformed immunoglobulin concentrations. Groups were compared using the Mann-Whitney U-test to establish the statistical significances between means. Correlations of individual values of the salivary level of SIgA and the serum level of specific IgE or total immunoglobulins were performed by the Spearman's test. A probability level of $<5 \%$ was considered to be statistically significant.

\section{Results}

\section{Serum levels of allergen-specific IgE}

Specific IgE was detected in the serum from all of the 20 JCP patients (lower detectable limit, $0.34 \mathrm{UA} / \mathrm{mL}$ ). The values ranged from 0.87 to $100<\mathrm{UA} / \mathrm{mL}$ with the median, $25^{\text {th }}-75^{\text {th }}$ percentile was $11.5 \mathrm{UA} / \mathrm{ml}, 4.05,23.1$, respectively. This shows that all JCP patients were CAP-RAST-positive to sensitized with the responsible allergen. On the other hand, 22 non-allergic control subjects were shown to be consisted of 14 CAP.RAST-negative (unsensitized) subjects and eight CAP·RAST-positive (sensitized) subjects. None of the unsensitized control subjects had a detectable concentration of specific IgE in the serum $(<0.34 \mathrm{UA} / \mathrm{mL})$, while all of the sensitized control subjects had detectable levels of specific IgE, ranging from 0.55 to $17.5 \mathrm{UA} / \mathrm{mL}$, with the mean value of $4.3 \pm 6.0 \mathrm{UA} / \mathrm{mL}$. As shown in figure 1, the mean specific IgE level in serum for all control subjects $(1.77 \pm 3.96 \mathrm{UA} / \mathrm{mL})$ was significantly lower than the value for JCP patients $(\mathrm{P}<0.001)$.

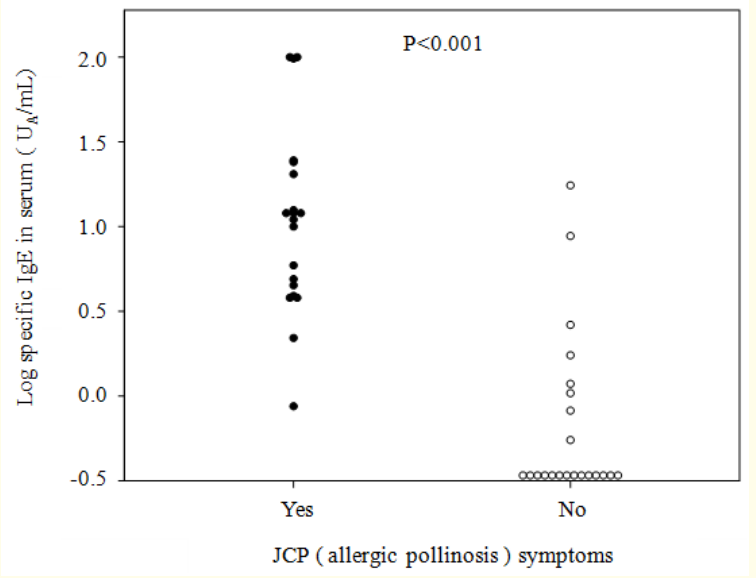

Figure 1: Serum levels of allergen-specific IgE in JCP patients ( $\mathrm{n}$ $=20)$ and non-allergic control subjects $(n=22)$. Values of $<0.34$ and $100<\mathrm{UA} / \mathrm{ml}$ were regarded as 0.34 and $100 \mathrm{UA} / \mathrm{ml}$, respectively.

\section{Salivary levels of SIgA}

Figure 2 shows individual values and the mean of the salivary level of SIgA for each of the JCP patient cohort and the control subject cohort. SIgA was detected in the saliva from all of the 27 study population (lower detectable limit, $0.06 \mu \mathrm{g} / \mathrm{mL}$ ). The values for the JCP group ranged from 39.0 to $71.5 \mu \mathrm{g} / \mathrm{mL}$ with the mean of 58.1 $\pm 8.8 \mu \mathrm{g} / \mathrm{mL}$, while those for the control ranged from 42.0 to 81.4 $\mu \mathrm{g} / \mathrm{mL}$ with the mean of $68.6 \pm 12.1 \mu \mathrm{g} / \mathrm{mL}$. There was a significant difference in the mean value of the SIgA level in saliva between the two cohort $(\mathrm{p}=0.003)$. The mean salivary SIgA level for sensitized subjects in the control subject cohort $(70.0 \pm 11.8 \mu \mathrm{g} / \mathrm{mL})$ was closely similar to the value for unsensitized subjects of this cohort $(67.9 \pm 12.6 \mu \mathrm{g} / \mathrm{mL})$, and was significantly higher than the value for the JCP patients $(\mathrm{p}=0.032)$. These results suggest the possibility that low salivary levels of SIgA may increase a risk for developing symptomatic JCP in the adult population, whether they are sensitized with the responsible allergen or not.

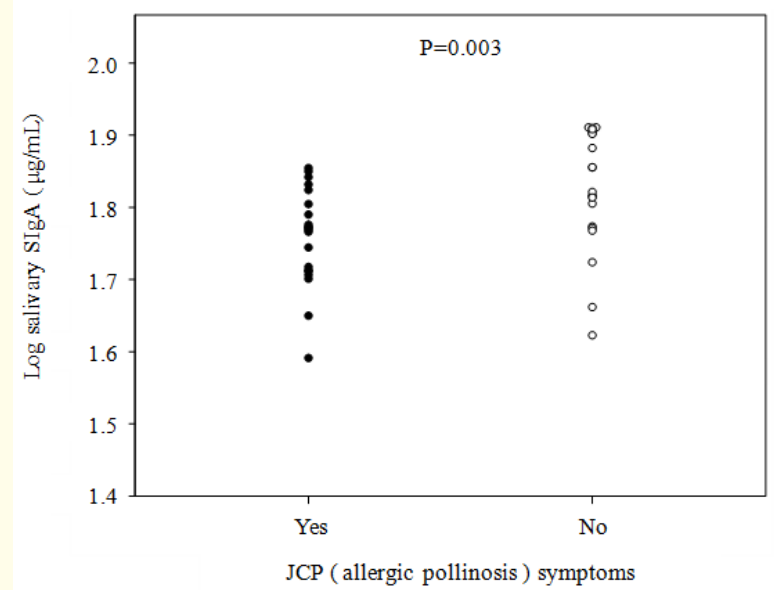

Figure 2: Salivary levels of SIgA in JCP patients $(n=20)$ and non-allergic control subjects $(n=22)$.

Correlation between salivary levels of SIgA and serum levels of allergen-specific IgE

As seen in figure 3, a significant inverse relationship was recognized between salivary levels of SIgA and serum levels of IgE specific for Japanese cedar pollen allergen in the whole study population $(\gamma=-0.34, p=0.029)$. In contrast, no significant correlation was observed between salivary SIgA levels and total serum levels of IgE, IgA or IgG (data not shown). 


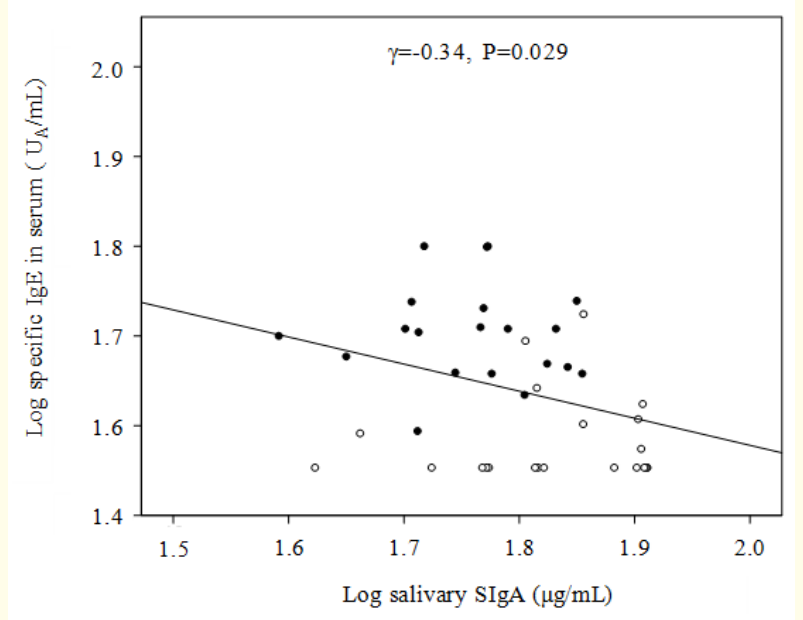

Figure 3: Inverse relationship between serum levels if specific IgE (vertical) and salivary levels of SIgA in the whole study population consisting of JCP patients ( $\mathrm{n}=20$ ) and non-allergic control subjects $(\Pi 7 ; n=22)$.

\section{Discussion and Conclusion}

It is shown from the present study that the mean salivary level of SIgA was significantly lower in a JCP patient cohort who had substantial serum levels of allergen-specific IgE than in an almost age-matched non-allergic control subject cohort with lower or undetectable levels of allergen-specific IgE. As a result, although there were considerable interindividual variations, salivary levels of SIgA were inversely correlated with serum levels of specific IgE to a statistically significant level. We also found that there was no significant difference in the mean salivary level of SIgA between the CAP-RAST-positive (sensitized) control subjects and the CAP-RAST-negative (unsensitized) control subjects. These results imply that development of symptomatic JCP but may be associated with low levels of SIgA in saliva and probably in other mucosal secretions, such as respiratory secretions and tears, no matter how the individuals were sensitized to specific antigen or not.

A number of papers thus far reported that there is a possible relationship between deficiency or low levels of SIgA and development of allergy in neonates or young children under 2 years of age $[8-10,11,15]$. However, contradictory results were obtained from almost all studies in older children [9,12,16-18] which have failed to demonstrate differences in SIgA levels between atopic and non-atopic infants. Although there is no rational explanation for the discrepancy of the results between these two different age population, the age-dependent change in secretory immunological status, in terms of local production and secretion of SIgA, might be involved.

Contrary to such a large number of infant studies that were conducted to examine SIgA levels in relation to the development of allergic disease, adult studies dealing with this issue are scarcely reported. Exceptionally, a Japanese research group demonstrated that both the amount of SIgA in sweats from a population of patients with atopic dermatitis (AD) (range, 13 to 44 years; mean, 22.9 years) and the value in tears from another study population of AD patients (range, 2 to 38 years; mean, 23.2 years) are significantly lower than the corresponding age-matched healthy controls $[19,20]$. In consistent with these studies, our study that was conducted in adult patients with JCP (range, 22 to 53 years; mean, 42.0 years), compared with sensitized and unsensitized non-allergic control adult individuals support the possible notion that low levels of SIgA in saliva or other mucosal secretions may involved in the development of allergy.

The present study also shows that among the sensitized (CAP-RAST-positive) adults, only those with low levels of SIgA in saliva have manifested allergic symptoms. Secretory IgA present in saliva and probably in other mucosal secretions binds various allergens to form complexes, preventing the allergens to adhere to and subsequently penetrate through the mucosal surface [21,22]. This may result in inhibition by a mechanism, which is referred as immune exclusion [22], of antigen-presentation to the immune system so that allergic inflammatory responses are not provoked. Total SIgA measured in our study is considered to include allergenspecific SIgA antibodies provided by the secretory immune system. Immunoglobulin A antibodies are capable of suppressing chemotaxis of neutrophils, eosinophils and monocytes [23] and inhibiting IgE-induced histamine-release [24]. Presumably, through both of non-specific and specific immunological functions of SIgA, high levels of SIgA in the mucosa could possibly interfere with not only the production of specific IgE but also its interaction with the allergen, thereby playing a protective role against the development of allergic diseases.

A number of factors are known to influence SIgA levels in saliva and other mucosal secretions. Among them is the local immunotherapy which tends to increase SIgA levels in salivary or nasal 
secretions $[8,15,25,26]$. It looks, therefore, likely that the effectiveness of immunotherapy to alleviate allergic symptoms might be due to not only a suppression of specific IgE production but also an enhancement of production and secretion of SIgA. Hence, induction of increased SIgA production in mucosal secretions can be considered as a promising strategy for the prevention and treatment of mucosal forms of allergy, including JCP.

In this regard, there are papers reporting that bovine colostrum supplementation increases salivary levels of total IgA or SIgA in athletes, who are under physical and/or psychological stresses, probably through improvement in mucosal immunity $[27,28]$. The mucosal immune system constitutes a common network and the B cells responsible for local production of SIgA are mainly derived from GALT, particularly Payer's patches [3], which render primed $B$ cells to migrate via peripheral blood to mucosa throughout the body including the salivary gland. It seems, therefore, possible that orally administered bovine colostrum may stimulate Payer's patches to promote SIgA production in saliva. Supplementary diets with such a stimulatory effect on mucosal immunity may have the potential for improving JCP and other allergic diseases.

\section{Bibliography}

1. Brandtzaeg P., et al. "Production and secretion of immunoglobulins in the gastrointestinal tract". Annals of Allergy 59 (1987): 21-39.

2. Brandtzaeg P. "The role of humoral mucosal immunity in the induction and maintenance of chronic airway infections". American Journal of Respiratory and Critical Care Medicine 151 (1995): 2081-2087.

3. Brandtzaeg P., et al. "Regional specialization in the mucosal immune system: what happens in the microcompartments?" Immunology Today 20 (1999): 141-151.

4. Johansen F., et al. "The J chain is essential for polymeric Ig receptor-mediated epithelial transport of IgA". Journal of Immunology 167 (2001): 5185-5192.

5. Soothill JF., et al. "Predisposing factors and the development of reaginic allergy in infancy". The Journal of Allergy and Clinical 6 (1976): 305-319.

6. Kilian M., et al. "Perturbation of mucosal immune defense mechanisms by bacterial IgA proteases". Bulletin Européen de Physiopathologie Respiratoire 19 (1983): 99-104.

7. Burks AW and Steele RW. "Selective IgA deficiency". Annals of
Allergy 57 (1986): 3-9.

8. Payette K and Weiss NS. "Salivary IgA levels in atopic children". Annals of Allergy 39 (1977): 328-331.

9. Van Asperen PP., et al. "The relationship between atopy and salivary IgA deficiency in infancy". Clinical and Experimental Immunology 62 (1985): 753-757.

10. Calvo M., et al. "Evaluation of secretory IgA in childhood respiratory diseases". Allergologia et Immunopathologia 16 (1988): 157-161.

11. Böttcher MF., et al. "Total and allergen-specific immunoglobulin A levels in saliva in relation to the development of allergy in infants up to 2 years of age". Clinical and Experimental Allergy 32 (2002): 1293-1298.

12. Gleeson M., et al. "Development of bronchial hyperreactivity following transient absence of salivary IgA". American Journal of Respiratory and Critical Care Medicine 153 (1996): 17851789.

13. Okuda M. "Epidemiology of Japanese cedar pollinosis throughout Japan". Annals of Allergy, Asthma and Immunology 91 (2003): 288-296.

14. Kohler PF and Farr RS. "Quantitative comparison of immunoglobulins in atopic (reaginic) and nonatopic (nonreaginic) individuals: Higher $\gamma \mathrm{D}$ levels in atopic sera". The Journal of Allergy 39 (1967): 311-322.

15. Plebani A., et al. "Different role of secretory IgA in the pathogenesis of RAST-positive and RAST-negative atopic dermatitis". Clin Allergy 12 (1982): 403-407.

16. Brasher GW. "Salivary IgA in children with atopic disease". Annals of Allergy 29 (1971): 422-427.

17. Hobday JD., et al. "A comparison of the immunoglobulins IgA, IgG and IgE in nasal secretions from normal and asthmatic children". Clinical and Experimental Immunology 9 (1971): 577-583.

18. Salvaggio J., et al. "Salivary, nasal wash and sputum IgA concentrations in atopic and non-atopic individuals". The Journal of Allergy and Clinical Immunology 51 (1973): 335-343.

19. Imayama S., et al. "Reduced secretion of IgA to skin surface of patients with atopic dermatitis". The Journal of Allergy and Clinical Immunology 94 (1994): 195-200. 
20. Toshitani A., et al. "Reduced amount of secretory component of IgA secretion in tears of patients with atopic dermatitis". The Journal of Dermatological Science 19 (1999): 134-138.

21. Taylor B., et al. "Transient IgA deficiency and pathogenesis of infantile atopy". Lancet 2 (1973): 111-113.

22. Stokes CR., et al. "Immune exclusion is a function of IgA". Nature 255 (1973): 745-746.

23. Brandtzaeg P. "Molecular and cellular aspects of the secretory immunoglobulin system". APMIS 103 (1995): 1-19.

24. Turk A., et al. "Nasal secretory antibody to inhalant allergens in allergic and non-allergic patients". Immunology 19 (1970): 85-95.

25. Georgitis JM., et al. "Local intranasal immunotherapy for grass-allergic rhinitis". The Journal of Allergy and Clinical Immunology 71 (1983): 71-76.

26. Gurgenidze GV., et al. "Comparative estimation of general and local humoral immunity indicators in connection with local immunotherapeutic treatment". Allergologia et Immunopathologia 18 (1990): 325-329.

27. Mero A., et al. "IGF-I, IgA, and IgG responses to bovine colostrum supplementation during training". Journal of Applied Physiology 93 (2002): 732-739.

28. Crooks CV., et al. "The effect of bovine colostrum supplementation on salivary IgA in distance runners". International Journal of Sport Nutrition and Exercise Metabolism 16 (2006): 47-64.

\section{Assets from publication with us}

- Prompt Acknowledgement after receiving the article

- Thorough Double blinded peer review

- Rapid Publication

- Issue of Publication Certificate

- High visibility of your Published work

Website: www.actascientific.com/

Submit Article: www.actascientific.com/submission.php

Email us: editor@actascientific.com

Contact us: +919182824667 